\title{
Ultra High Performance Media Multicasting Scheme over Wavelength-Routed Networks
}

\author{
Malek Ghandour ${ }^{1}$, Sheng Liu ${ }^{2}$, David K. Hunter ${ }^{1}$, Dimitra Simeonidou ${ }^{1}$, Reza Nejabati ${ }^{1}$, Periklis Petropoulos ${ }^{2}$ \\ 1-Networked Media Laboratory, University of Essex.Colchester, CO4 3SQ.UK, Email: mghand@essex.ac.uk \\ 2-Optoelectronics Research Centre, University of Southampton. Southampton, UK.
}

\begin{abstract}
This paper presents a demonstration of an all-optical multicasting scheme for ultra high definition media over wavelength-routed networks, using Self-Phase Modulation. The feasibility of using this technique has been experimentally and theoretically evaluated for streaming rates up to $100 \mathrm{Gbps}$.

(C)2010 Optical Society of America

OCIS codes: (0604255) Networks, multicast; (0602330) Fiber Optics Communications
\end{abstract}

\section{Introduction}

Next generation high-performance multimedia services will include super and ultra high resolution video formats ( $4 \mathrm{~K}$ and $8 \mathrm{~K}$ ). Such formats will require single view data rates varying from $384 \mathrm{Mbps}$ up to 24 Gbps [1]. The development of high-performance optical networks comprising wavelength switching, with Generalized MultiProtocol Label Switching (GMPLS) capability, will make ultra high definition digital media (UHDM) distribution possible over optical networks. Typically, UHDM services require long-lived high capacity connectivity between a limited number of well known users and data resources. Lambda switching could therefore be the technology of choice for this application, guaranteeing that high volume digital streams are securely delivered - from source to destination - with the required QoS by these applications.

Multicasting avoids the need for duplication of information transmission over any one link, which minimizes transceiver usage in the network, and reduces the overall number of wavelengths required. By extending multicasting into the optical domain, multimedia applications will be carried over high-capacity circuits. For content distribution, it is particularly important that this approach will offer reduced jitter and reduced delay while avoiding (or at least ameliorating) the problems inherent in layer 3 (L3) solutions which exhibit datagram loss, and reducing the equipment cost required to achieve a given level of performance.

Table 1: Multicast requirements for UHDM [1]

\begin{tabular}{|c|c|c|c|c|}
\hline & $\begin{array}{c}4 K \\
\text { Compressed }\end{array}$ & $\begin{array}{c}8 K \\
\text { Compressed }\end{array}$ & $\begin{array}{c}\text { 4K } \\
\text { Uncompressed }\end{array}$ & $\begin{array}{c}8 K \\
\text { Uncompressed }\end{array}$ \\
\hline Bit rate & 382Mbps & 1.2Gbps & 7.64Gbps & 24Gbps \\
\hline Transmission System & Satellite & Optical & Optical & Optical \\
\hline Multicast Protocol & $\begin{array}{c}\text { L3 } \\
\text { (Electronic) }\end{array}$ & $\begin{array}{c}\text { L3 } \\
\text { (Electronic) }\end{array}$ & $\begin{array}{c}\text { L1 } \\
\text { (Optical) }\end{array}$ & $\begin{array}{c}\text { L1 } \\
\text { (Optical) }\end{array}$ \\
\hline
\end{tabular}

In this paper, investigation into the use of a modified configuration of Self Phase Modulation, using a highly nonlinear fibre (HNLF), is experimentally demonstrated for the purpose of providing regenerative WDM multicasting functionality. The technique presents several advantages for the particular application. First, multiple wavelength conversion and $2 \mathrm{R}$ regeneration of the original signal are achieved simultaneously. In addition, the HNLF provides environmental stability as well as simplicity of operation. The technique eliminates the need for additional laser sources. It uses only a short length of fibre (of the order of hundreds of meters), and has weak polarization dependence.

Optical multicasting can be achieved by applying this method for the data plane at every multicast node in a preestablished lightpath tree. When setting up multicast paths, a wavelength - between two nodes - with acceptable BER performance will be chosen from the available wavelengths of the replicas generated at the multicast node.

\section{Principle of Multi-Wavelength Conversion using SPM and Experimental Setup}

Multi-Wavelength Conversion (MWC), is an attractive technology for producing multiple copies of the multicast stream directly in the optical domain. Self-Phase Modulation (SPM) in an HNLF has shown ability for 


\section{JThA40.pdf}

MWC in conjunction with an arrayed waveguide grating (AWG) [2]. When a data signal propagates in an HNLF, its spectrum is broadened due to SPM. The spreading of the spectrum depends on the choice of the fibre nonlinear coefficient, dispersion and dispersion slope, as well as the signal input power. Subsequent optical filtering, at wavelengths that are shifted with respect to the incoming wavelength, will generate replicas of the input signal, which can actually be reshaped in amplitude relative to the input to remove any amplitude fluctuations.

By appropriately choosing the central wavelengths and the AWG parameters, multiple WDM channels are formed. The original data signal is replicated into these channels, i.e. WDM multicasting is performed. By ensuring a flat SPM spectrum over the bandwidth of interest, only small variations in the powers of the various channels will be achieved.

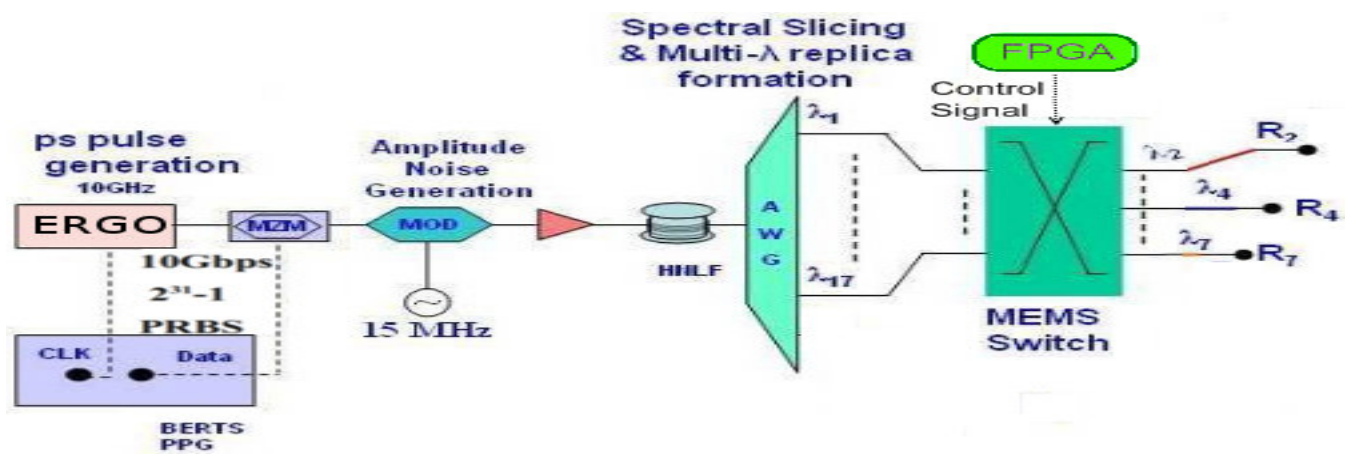

Figure 1: $10 \mathrm{~Gb} / \mathrm{s}$ Experimental Block Diagram

Since the nonlinear behaviour of the HNLF originates from the Kerr effect, its nonlinear response is practically instantaneous, making this kind of regenerator particularly suitable for future high data rate systems.

The generation of the replicas is ultra-fast; at the output of the AWG, the only delay is due to chirp which is of the order of tens or hundreds of pico-seconds. This delay will be overshadowed by the subsequent switching delay.

Table 2: HNLF parameters measured at 1550nm (source: Furukawa Electric Co)

\begin{tabular}{|c|c|c|c|c|c|c|}
\hline Fibre & $\begin{array}{c}\text { Attenuation } \\
(\mathrm{dB} / \mathrm{km})\end{array}$ & $\begin{array}{c}\text { Dispersion } \\
(\mathrm{ps} / \mathrm{nm} / \mathrm{km})\end{array}$ & $\begin{array}{c}\text { Slope } \\
\left(\mathrm{ps} / \mathrm{nm}^{2} / \mathrm{km}\right)\end{array}$ & $\begin{array}{c}\gamma \\
\left(\mathrm{W}^{-1} \cdot \mathrm{km}^{-1}\right)\end{array}$ & $\begin{array}{c}\text { PMD } \\
\left(\mathrm{ps} / \mathrm{km}^{1 / 2}\right)\end{array}$ & $\begin{array}{c}\text { Length } \\
(\mathrm{m})\end{array}$ \\
\hline HNLF-DSF2 & $\mathbf{0 . 4 9}$ & $\mathbf{- 0 . 6 4}$ & $\mathbf{0 . 0 3}$ & $\mathbf{2 0}$ & $\mathbf{0 . 0 5}$ & $\mathbf{4 9 0}$ \\
\hline
\end{tabular}

An experiment was set up at $10 \mathrm{Gbps}$, Figure 1, in order to prove the principle of operation. The laser source used was a $10 \mathrm{GHz}$ Erbium Glass Oscillator (ERGO) pulse source with 2ps-pulses at a wavelength of $1546 \mathrm{~nm}$. Amplitude noise was intentionally introduced to the pulses in a controlled fashion using a modulator, which was driven by a $15 \mathrm{MHz}$ sinusoidal signal. Using this approach, it was possible to add intensity noise to the 'zero' and/or the 'one' levels of the input pulses in a deterministic way, which permitted assessment of the performance of the regenerator in terms of amplitude equalization. The parameters of the HNLF used are tabulated above (Table 2).

\section{Results}
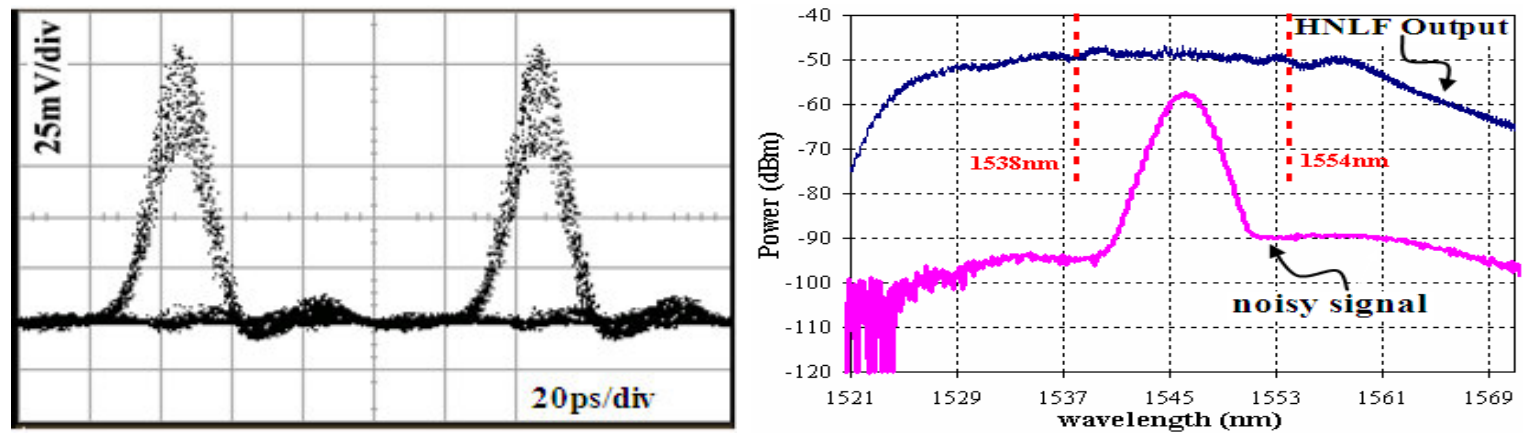

Figure 2 a) The eye-diagram of the noisy input signal, b) the spectrums of the noisy signal and the output of the HNLF 
The output power of the Erbium doped Fibre Amplifier was set to $+19 \mathrm{dBm}$ which ensured that enough broadening was achieved. The eye-diagram of the noisy input signal to the HNLF, and the spectra of the noisy signal and the output of the HNLF are shown in Figures 2(a) and 2(b) respectively. A range of $16 \mathrm{~nm}(1538-1554 \mathrm{~nm})$ is studied to determine the replicas generated, where the output power varied from $-3.4 \mathrm{dBm}$ at $1546 \mathrm{~nm}$ to $-6.25 \mathrm{dBm}$ at $1551.6 \mathrm{~nm}$. The AWG has $0.62 \mathrm{~nm} 3-\mathrm{dB}$ bandwidth and $0.8 \mathrm{~nm}$ channel spacing. Figure 3(a) shows the output eyediagram at $1544.4 \mathrm{~nm}$.
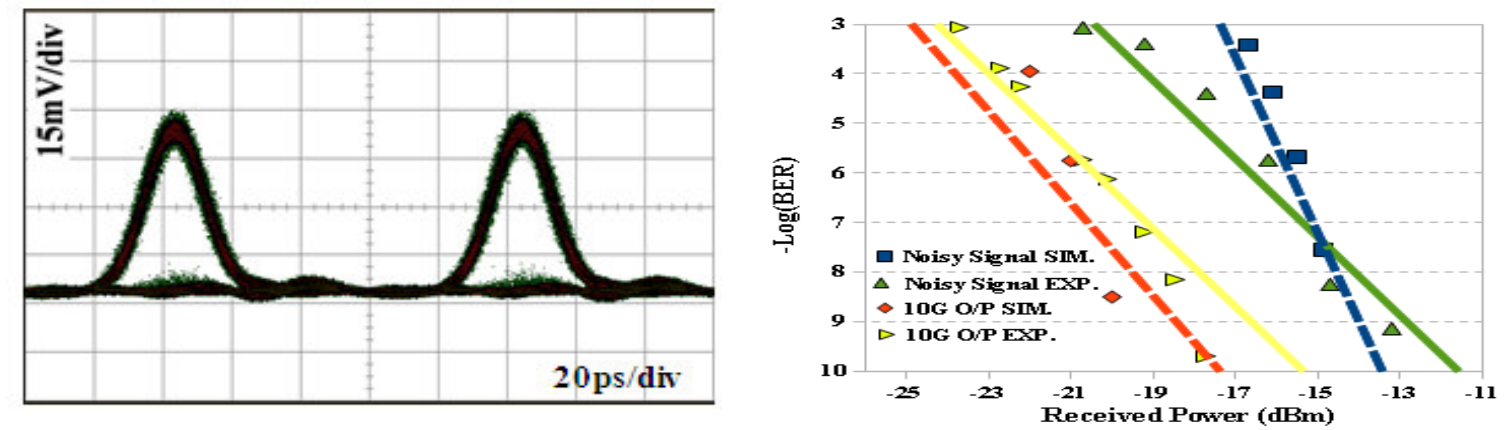

Figure 3 a) the eye-diagram of a replica at $\lambda=1544.4 \mathrm{~nm}$ b) $10 \mathrm{Gbps}$ BER performance with $24 \%$ Amplitude Noise (Simulation \& Experimental)

A 10Gbps Bit Error Rate Tester (BERT) was used to measure the BER of several replicas, with respect to the received power or at a fixed power level and different added noise levels. Figure 3(b) compares the BER performance of an incoming signal with $24 \%$ induced amplitude noise with that of the output at $1544.5 \mathrm{~nm}$, measured experimentally and by simulation (using VPI version 7.6). A 4dB improvement in receiver sensitivity at BER of $10^{-9}$ is observed. Figure 4(a) shows the BER performance of all the outputs of the AWG in the 16nm range selected, measured at a received power of $-20.11 \mathbf{~ d B m}$, with different added noise levels of $4 \%, 12.5 \%, 24 \%$ of the amplitude of the 'one'. Figure 4(a) also shows that at least 14 replica outputs with a minimum of $4 \mathrm{~dB}$ improvement in the sensitivity of the receiver can be considered for Optical Multicast using this technique.
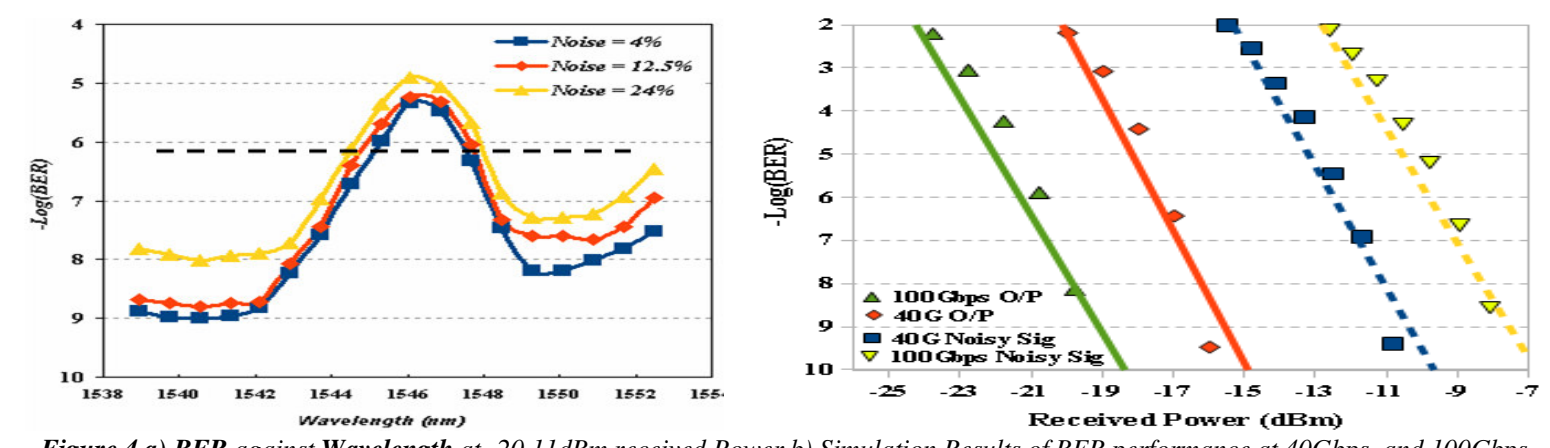

Figure 4 a) BER against Wavelength at -20.11 dBm received Power b) Simulation Results of BER performance at 40Gbps, and 100Gbps

\section{Conclusion}

In this paper, we have demonstrated experimentally at $10 \mathrm{Gbps}$ the potential of SPM-based spectral slicing for UHDM multicasting. We specifically focused on the $16 \mathrm{~nm}$ span around the central wavelength, and achieved 14 regenerated replicas of a 10Gbps UHDM stream; however even more replicas can be generated outside this range. We have also provided simulation results for 40 and 100Gbps (figure 4(b)) in order to demonstrate the feasibility of this technique at high-data-rate UHD media multicasting.

Acknowledgment: The work described in this paper was carried out with the support of the BONE-project ("Building the Future Optical Network in Europe"), a Network of Excellence funded by the European Commission through the 7th ICT-Framework Programme.

\section{References}

[1] D. Simeonidou et al., "Optical Network Services for Ultra High Definition Digital Media Distribution” IEEE GOSP 2008, London, United Kingdom, Sept 2008

[2] F. Futami and S. Watanabe, "All-optical Wavelength Conversion Using Ultra-Fast Nonlinearities in Optical Fiber," IEICE Trans. Electron., vol. E85-C, pp. 889-894, 2002. 